\title{
Pools: a More Efficient Way to Support Spacecraft Operations
}

\author{
A. Codazzi ${ }^{*}$ and M. $\mathrm{Kim}^{\dagger}$ \\ German Aerospace Center, Munich, Germany, 82234
}

\begin{abstract}
The operations department at the GSOC recently started using a new approach for the support of satellite missions. Since 2009, several pools of specialists have been created, that cover all missions instead of having dedicated teams for each project. Each pool groups experts of the particular satellite subsystems (e.g. attitude and orbit control, power/thermal, data, etc.). All pools together form the Operations Engineering Group (OEG), which is managed by the OEG lead, and whose main goal is to satisfy the project needs, using group resources in the most efficient way. To achieve this objective the group lead together with the individual group members, are constantly trying to improve the group performance by optimizing not only the internal structure and the exchange of information with the project managers (the so-called "hard" factors), but also by working on the "soft" factors. The latter are, for example, assigning tasks to the engineers, keeping an eye on employee motivation, communicating inside the pools and the cross-communication between them, encouraging employee creativity, and establishing a group culture. This paper focuses on the OEG's main features. It describes the experiences of the last two years supporting an increasing number of projects using the described approach. The first section gives a general overview of the group. The advantages of supporting operations with different pools instead of using dedicated teams are presented in Section 2. Section 3 describes the challenges faced by the group and the solution applied. The fourth section presents some conclusions, the lessons learned and some possible future applications of this promising support strategy.
\end{abstract}

\section{Introduction}

$\mathrm{T}$

HE DLR (Deutsches Zentrum für Luft- und Raumfahrt) is Germany's national research centre for aeronautic and space with main research fields in aeronautics, space, transportation and energy. There are a total of 33 institutes and facilities all over Germany with a total work force of approximately 6900. Spacecraft mission operations are carried out in one of these institutes: the German Space Operations Center (GSOC), located in Oberpfaffenhofen. At the GSOC, the "mission operations" department performs the project's operational tasks.

This department can be divided into three main sub-units. The first focuses on manned missions, the second takes care of communication satellites, and the third supports technology and Earth observation projects. The latter in turn consists of three groups: the projects management group (PMG), the software engineering group (SEG), and the operations engineering group (OEG).

The OEG supports all the operations engineering related tasks, as defined by the ECSS standards ${ }^{1}$ :

1) During phase A (mission and operational analysis, feasibility study and conceptual design), the OEG contributes to the mission requirements analysis and to the concept development. It supports the definition of the operational aspects of the mission concept at the space system level. Using the experience of over 40 years in spacecraft operations, the OEG can analyze subsystem related tasks, contributing to the project effort estimation.

2) During phase B (preliminary design), the OEG supports the specification of the ground systems requirements. It contributes to the operational analysis and to the development of the operational concept.

\footnotetext{
* Operations Engineering Group Leader, Mission Operations Department, Alessandro.Codazzi@dlr.de

† Operations Engineer, Mission Operations Department, Mischa.Kim@dlr.de
} 
3) During phase C (detailed design), the OEG contributes to the mission operations plan.

4) During phase $D$ (production, assembly, integration, test and verification), the OEG supports the system integration and its operational validation. The group's engineers develop and validate the flight operations procedures starting from the command sequences delivered by the spacecraft manufacturer. The operations engineers also prepare the displays that are used for monitoring spacecraft telemetry. Furthermore, the group takes care of the administration, the monitoring and the maintenance of the spacecraft "failure detection, isolation and recovery" routines. The preparation of training and simulation sessions and the participation in those sessions is also an OEG task.

5) During phase E (mission operations), the OEG contributes to the planning of subsystem operations. The group supports the subsystem operations during LEOP, the commissioning and the routine phases. The group engineers analyze the performance of the spacecraft subsystems and handle anomalies. The OEG delivers also an on-call support, and takes care of the subsystem configuration management.

6) During phase F (disposal), the group supports the preparation of mission termination and the space segment disposal operations.

This paper focuses on the operations engineering group (OEG) and the measures applied to fully capitalize on its human capital. It is described how, in order to reach this goal, the OEG works not only on the organizational structure and on the processes applied to carry out the daily business (the so-called "hard" factors), but considered also the so-called "soft factors". These include leadership styles, the shared values, and the actual skills and competencies of the group members.

Typically, "hard" elements are easier to define and management can directly influence them. "Soft" elements, on the other hand, can be more difficult to describe, are less tangible, and more influenced by the group culture. Nevertheless, there is clear evidence that shows that these soft elements are as important as the hard elements for the success of an organization ${ }^{2}$.

In the following chapter, the multi-mission support strategy applied by the mission operations department is introduced.

\section{The "pools" strategy}

The resources necessary to support a project throughout its lifetime vary. The typical project's course is sketched in Fig. $1^{\ddagger}$.

Workload fluctuations are normal for any project, especially those creating "something new" ${ }^{3}$. This is the case with most spacecraft missions.

More of than not, spacecraft are designed and built as prototypes. Therefore, the operations systems often have to be built from scratch or, in the best case, need to be strongly adapted.

Spacecraft missions present further challenges for the managers in charge. For example, before the start of the mission, launch delays, difficulties in meeting the delivery deadlines and other factors can strongly affect the project schedule. After launch, anomalies and contingencies support and recovery constitute workload peaks that are impossible to be foreseen.

Efficiency in coping with such

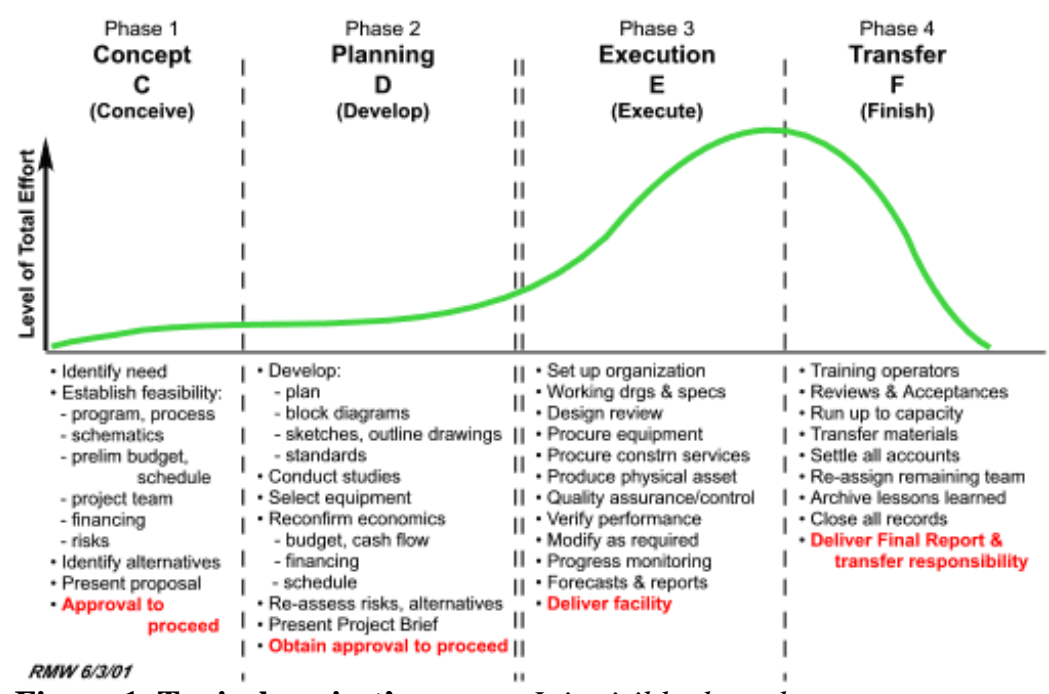

Figure 1. Typical project's course. It is visible, how the resources necessary to support a project vary throughout its lifetime.

variable support need is a very important factor for company success. Therefore, team flexibility and scalability are core assets when dealing with spacecraft operations.

\footnotetext{
${ }^{\ddagger}$ http://www.maxwideman.com
} 


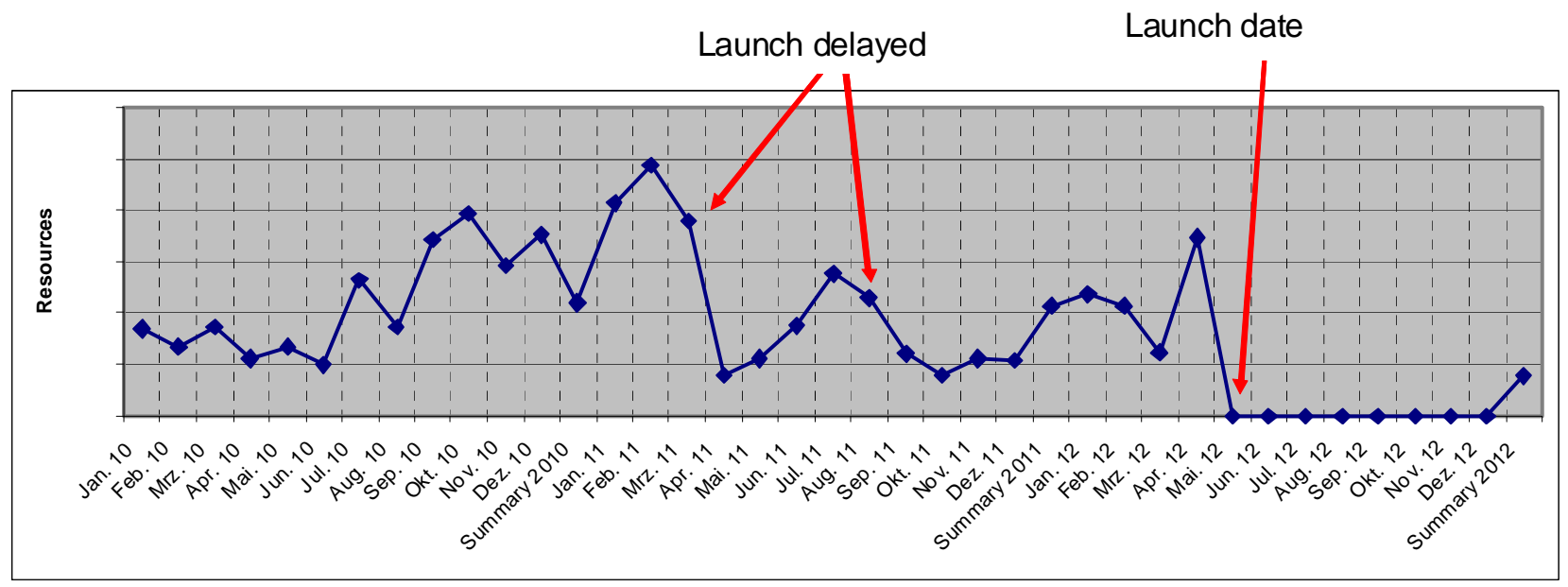

Figure 2. Consequences of launch delays on the resources utilization in the period from Jan. 2010 and Apr.

2012. The support needed by the project increases as the launch comes closer. The launch delay causes a drop.

Typically, a pause is introduced until the next launch preparation phase.

Formerly, the GSOC mission operations department supported each mission with a dedicated team. As the number of missions increased, the department faced a challenge: with the available resources, this strategy could no longer be supported. Instead of hiring new operations engineers, the decision was taken to create a structure that by coordinating the sharing of resources between the projects - would have allowed a more efficient utilization of the personnel.

In 2009, three groups of engineers were created considering the department's core competencies: the software engineering group (SEG), taking care of the development and implementation of software solutions, the project management group (PMG), grouping the managers of the spacecraft missions operations, and the operations engineering group (OEG). A lead was assigned to each group.

The SEG and OEG leads were assigned with the task of controlling project efforts with an adequate resources allocation plan to be able to keep project managers informed and to make suitable adjustments as necessary.

The three groups were supposed to closely cooperate, share information, tasks and some of the resources. For this purpose, intense communication between the group leads was encouraged by the department management from the introduction of the pool concept.

The next chapter focuses on the feature of the OEG.

\section{The Operations Engineering Group (OEG)}

Approximately 25 engineers are supporting the OEG. Of those, roughly $40 \%$ are core members, $15 \%$ are contractors, and the remaining $45 \%$ are engineers from the other groups dedicating part of their time to operations engineering. In total, the OEG can provide a workforce roughly 17 men strong. $80 \%$ of those are DLR employees, $20 \%$ are contractors.

The OEG is divided into four sub-groups according to the core competencies: attitude and orbit control (AOCS), on-board computer, telemetry and telecommand (OBC/TMTC), power and thermal (PTS), and operations system engineering. The first three correspond to the main subsystems of a spacecraft (excluding the payload), the fourth is mainly related to coordination tasks and establishment of interfaces with other systems not operated by the OEG (for example the mission planning system, the flight dynamic system, or the payload calibration system).

Each sub-group has a coordinator, who is the prime point of contact the OEG lead refers to for sub-group related issues. In addition, the sub-group coordinators support the OEG lead in planning tasks assignments, estimating the projects' related efforts, evaluating employee performance, training and developing of (new) employees, and staffing. However, the group coordinator spends the larger part of the nominal working day providing technical support to the missions.

Thanks to the support of the sub-group coordinator in fulfilling the management tasks, the OEG lead can focus on the leadership related aspects. More details will be given in the following chapters. 


\section{The Challenges}

The McKinsey 7s model states that for a change to be successful there are seven elements that need to be adjusted (or re-adjusted). These elements, divided into "hard" (strategy, skills, structure) and "soft" (systems, staff, style, shared values) factors, are mutually interconnected.

The establishment of the pool concept was a significant change in the GSOC mission operations department. New players (i.e., the group leads) in charge of allocating scarce resources have been introduced in the organizational structure. Established routines, for example those related to the assignment of resources, have been challenged. As a consequence of the strategy change, the operations engineers moved to a multi-mission environment, where most of them had to support several projects in parallel.

A survey conducted in large German companies showed that only $20 \%$ of the changes undertaken fully succeeded in reaching their objectives. The results were used to create an analytical model for explaining successful change. The model identifies four key success factors: leadership, experience in coping with uncertainties, motivation and congruence ${ }^{2}$.

The leadership factor consists of the top-management

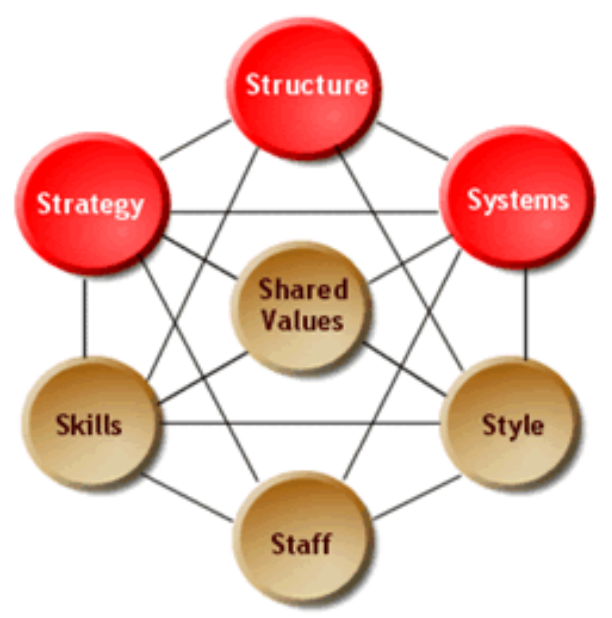

Figure 3. The McKinsey 7s model. "Hard" and "soft" factors, together with their interconnections, are displayed. engagement, the clarity of the objectives, the consensus on top-management level and the support of the middle management.

The experience in coping with uncertainties has to be seen from different perspectives. On one side, the employees must have enough expertise to understand and cope with uncertainty. On the other side, managers have to be able to carefully consider the employees' feelings, and intervene whenever necessary.

The actors' motivation is a factor that can strongly affect the change process results. The more the employees are motivated to support the change, the higher the probability of success. According to ${ }^{4}$, almost half of the employees withdraw support for the change process. In many cases, they resist or even fight it. Resistance to a change is not always explicit and easy to detect. The behaviors aimed at avoiding the change process, without giving the impression of resisting to it can be grouped under the name "BOHICA" which translates to "Bend Over, Here It Comes Again" (the wind of change). BOHICA is in fact the most common behavior in changes that overstrain the employees.

According to the literature, a change has to take into account as many organizational aspects as possible, thus increasing the congruency of the process. The more factors are considered when performing a change, the higher the probability of success. Often, the change agents (i.e., the employees directing the change) concentrate on the "hard" factors, leaving the "soft" unchanged. In this respect it is worth to stress the importance of the organizational culture: according to ${ }^{5,6,7}$ the most frequently cited reason for change process failure is a neglect of the organizational culture.

\section{A. From hierarchical to matrix}

The department structure changed from a hierarchical one to a matrix structure. In the hierarchical structure, every operations engineer was subordinated to a single project manager. In the matrix, all operations engineers report to the OEG leader; at the same time, they are assigned to different projects and therefore report also to the corresponding project managers.

The OEG serves as the interface to coordinate the allocation of the operations engineers. The project managers, who do no longer have direct access to the resources, may perceive this change as working against their interests. In fact, the OEG is allowed to codetermine the amount of resources to be assigned to the project, with possible negative side effects on project results and achievements.

A feeling of uncertainty may arise, triggering actions that can be interpreted as resistance to the enforcement of the pools strategy. However, to resist loss of status, loss of pay, or loss of comfort, are not the same as resisting change $e^{4}$.

The OEG lead decided to use an "adaption strategy" instead of an "overcoming strategy" and scheduled a number of meetings with the different project managers. The purpose was to listen to and understand their concerns, adapting the OEG goals, plans and features as a consequence. The OEG group lead did not expect the project 
managers to be enthusiastic about the new strategy, since it reduced their influence on the resources allocation. Instead, the group lead focused on getting the project managers acceptance, defined as "approval despite diverging interests". In fact, the critical factor for the success is the acceptance of the change process, not the enthusiasm ${ }^{4}$.

To properly distribute the tasks between the group lead and the project manager, therefore avoiding possible conflicts, it is important to understand the difference between "management" and "leadership". James Kotterman suggests, that in a company these two terms are often used interchangeably, thus creating confusion $^{9}$.

In the literature, "Leadership" is defined as a "process of social influence in which one person can enlist the aid and support of others in the accomplishment of a common task", whereas "Management" is the "act of getting people together to accomplish desired goals and objectives using available

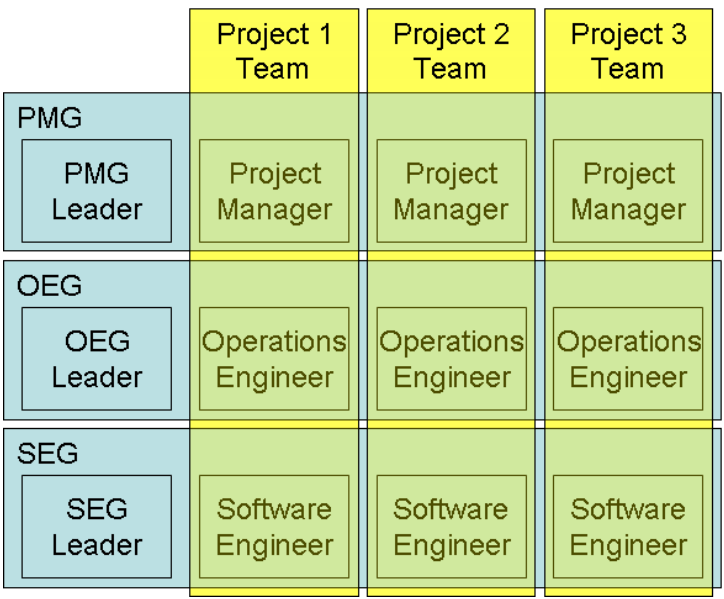

Figure 4: The department matrix structure. resources efficiently and effectively"§.

Kotterman states that "there is a general acceptance that the functions of leaders and managers are conceptually different, but no universal acceptance of what those functional differences are is apparent”. In his work, he compares leadership and management as shown in Table 1.

\begin{tabular}{|c|c|c|}
\hline Process & Management & Leadership \\
\hline $\begin{array}{l}\text { Vision } \\
\text { Establishment }\end{array}$ & $\begin{array}{l}\text { - Plans and budgets } \\
\text { - Develops process steps and sets timelines } \\
\text { - Displays impersonal attitude about the } \\
\text { vision and goals }\end{array}$ & $\begin{array}{l}\text { - Sets the direction and develops the vision } \\
\text { - Develops strategic plans to achieve the vision } \\
\text { - Displays very passionate attitude about the } \\
\text { vision } \\
\text { and goals }\end{array}$ \\
\hline $\begin{array}{l}\text { Human } \\
\text { Development } \\
\text { and Networking }\end{array}$ & $\begin{array}{l}\text { - Organizes and staffs } \\
\text { - Maintains structure } \\
\text { - Delegates responsibility } \\
\text { - Delegates authority } \\
\text { - Implements the vision } \\
\text { - Establishes policy and procedures to } \\
\text { implement vision } \\
\text { - Displays low emotion } \\
\text { - Limits employee choices }\end{array}$ & $\begin{array}{l}\text { - Aligns organization } \\
\text { - Communicates the vision, mission, and } \\
\text { direction } \\
\text { - Influences creation of coalitions, teams, and } \\
\text { partnerships that understand and accept the } \\
\text { vision } \\
\text { - Displays driven, high emotion } \\
\text { - Increases choices }\end{array}$ \\
\hline Vision Execution & $\begin{array}{l}\text { - Controls processes } \\
\text { - Identifies problems } \\
\text { - Solves problems } \\
\text { - Monitors results } \\
\text {-Takes low-risk approach to problem solving }\end{array}$ & $\begin{array}{l}\text { - Motivates and inspires } \\
\text { - Energizes employees to overcome barriers to } \\
\text { change } \\
\text { - Satisfies basic human needs } \\
\text { - Takes high-risk approach to problem solving }\end{array}$ \\
\hline Vision Outcome & $\begin{array}{l}\text { - Manages vision order and predictability } \\
\text { - Provides expected results consistently to } \\
\text { leadership and other stakeholders }\end{array}$ & $\begin{array}{l}\text { - Promotes useful and dramatic changes, such } \\
\text { as new products or approaches to improving } \\
\text { labour relations }\end{array}$ \\
\hline
\end{tabular}

Table 1. Comparison of management and leadership process differences in the workplace ${ }^{9}$.

In the end, the input from the project managers turned out to be very constructive and useful. The role of the group lead and project managers, and their respective tasks, were discussed and better defined. The skepticism of the project managers progressively disappeared as they felt their input was considered and integrated in the pool's concept.

It was agreed that the OEG lead has to make sure that the employees within the group recognize the value of the supported projects, and has to motivate them to actively engage in it. The OEG lead takes also care of aspects such as networking between the group members, creativity and innovation management, employees' career development,

$\S$ http://www.radford.edu 
(work) culture, and feedback. The assignment of project related tasks, the decision about priorities inside the project, and the planning of project activities are under the responsibility of the project manager.

In the area of allocating resources, where the competencies of the group lead and the project manager overlap, the following process is enforced: the project manager is free to define not only the support of project needs, but also the amount of personnel and staffing assignments. The function of the OEG lead is to satisfy and coordinate the requests coming from the different projects. In case of conflicts, an alternative is suggested, that has to be approved by the project manager in anyway. The OEG lead does not force the project to accept a solution. Potential conflicts between the projects are discussed and solved with the department lead.

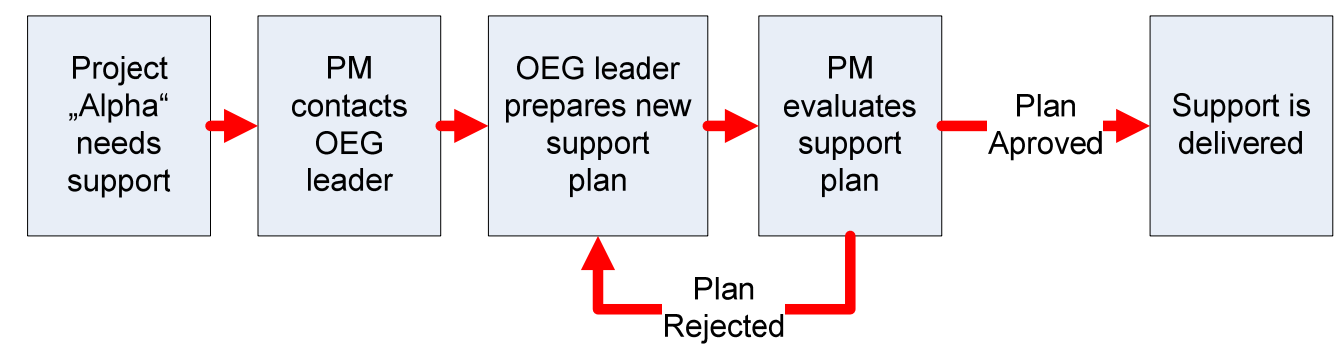

Figure 5: The process of allocating the operations engineering resources, as agreed by the OEG lead and the project managers

The task of controlling is assigned to the group lead, in order to allow a better comparison between the projects. Thanks to a controlling system based on monthly reports, the OEG lead is able to give feedback to the project manager about the amount of resources spent in supporting the project's main activities. In case of discrepancies between the plan and reality, a dedicated planning-update meeting is called by the OEG lead.

In general, it was agreed that the project related tasks have a higher priority than those related to the OEG, that are normally not associated with fixed milestones or delivery dates. Priorities between the projects are assigned by the department lead.

The discussions between the OEG lead and the project managers helped to establish an intense and open communication which became one of the crucial factors of the success of the pool concept.

\section{B. Loyalty and identification}

With the former strategy, each project was supported by a dedicated team. The current multi-mission concept implies that the operations engineers often support several projects in parallel. However, some questions arise: can a person be loyal to more than one project? Can an employee identify with the different missions supported?

"Loyalty" can be defined as "the willing and practical and thoroughgoing devotion of a person to a cause" 10 . Scholars observed that, in the general case, multiple loyalties do not cause a disloyalty. This would imply that an operations engineer can be loyal to different projects at the same time.

"Identification" (e.g. with a company or a team) is defined as "an active process by which individuals link themselves to elements in a social scene"12. Organizational identification can also be viewed as an alignment of individual and organizational values ${ }^{13}$. As a consequence, the decision makers desire to choose the alternative which best promotes the perceived interests of the organization ${ }^{14}$. Organizational identification is an important aspect because of its relationship with commitment to the organization ${ }^{15}$, which in turn positively affects motivation, job performance, job satisfaction, employee interaction and retention (with positive effects on productivity, efficiency, effectiveness), and individual decision making ${ }^{16}$.

Project managers often have very different characteristics, talents, and values, which influence their management style. Furthermore, the values of the project often reflect those of its manager. Therefore, each project team may develop its own "culture".

"Organizational culture" refers to the "taken-for-granted values, underlying assumptions, expectations, collective memories and definitions present in a company. It reflects what an employee expects from others, and constitutes the guidelines to interpret a particular situation"17.

Since the OEG members often work for more projects at the same time, they had to be able to adapt to and ideally identify (e.g., align their values) with different projects marked by different sets of values. The issue was discussed, and it has been concluded that, while adaptation is possible, identification might become problematic. This discussion also leads to another very important aspect: the OEG culture harmonization. 
The OEG members agreed that a common culture would improve the efficiency of the group. In fact, a common culture provides an "unproblematic background consensus". In cross-cultural communication, this background is reduced by an amount which is proportional to the cultural differences. Scholars believe that difficulties in coordinating and integrating processes or organizational activities, for example, are often a result of culture clashes among different subunits ${ }^{17}$.

Furthermore, by establishing a set of values, rules and beliefs everyone in the group did agree on, the group members were provided with a guideline to identify with, helping therefore their performance independently of the culture of the projects they were working on. The diagnosis of the organizational culture was made according to literature ${ }^{17}$.

As a first step, a survey was provided to the engineers. The results were used as a starting point for several meetings involving all OEG members. The purpose of the meetings was to talk about the current cultural situation, the goals, and the measures to be applied to achieve them. This resulted in the "OEG culture" text, which is included in the annex.

The establishment of the group culture has some side-effects that have to be carefully considered. In fact, a strong group culture may emphasize subunit cultural differences, fostering alienation and conflict. For the particular case described in this paper, this would mean clashes between the OEG and the other groups, or conflicts between operations engineers and project managers. To guard against those effects, the OEG group lead constantly encouraged flexibility in dealing with different cultures and encouraged the communication with employees outside the OEG.

\section{Motivation}

Employee motivation is considered to be a crucial factor for the success of a company. Several scholars engage themselves in understanding the triggers of motivation.

In his "acquired-needs" theory ${ }^{18}$, David McClelland proposed that individual needs develop over time, and are shaped by life experiences. According to McClelland, most of the needs can be classified as either "achievements", "affiliation", or "power". Motivation is influenced by these three ingredients. The degree, to which one need prevails over the others, and the way to motivate as a consequence, is assumed to be subject-dependent.

Herzber's two-factor theory ${ }^{19}$ states that there are aspects in the workplace that result in job satisfaction. They can be distinguished between "motivators" and "hygiene factors". Examples of motivators are responsibility, recognition or the possibility to work on challenging tasks. The hygiene factors are not motivators, but results in demotivation if not present. Examples of hygiene factors are salary and job security.

Steven Reiss, starting from the results of previous studies involving more than six thousands people, proposed a list of sixteen basic desires that motivate all human being's actions ${ }^{20}$. Among those are: curiosity (desire to learn), independence (need for individuality), order (need for organized and predictable environment), power (need for influence of will), and status (the need for social standing). Again, the degree to which one need prevails over the others depends on the employee's characteristics.

The goal of the OEG lead is to enhance the intrinsic motivation of the group members capitalizing on the possibilities offered by the pools strategy. Intrinsic motivation refers to motivation that is driven by an interest or enjoyment in the task itself, and is based on taking pleasure in an activity rather than working towards an external reward $^{* *}$.

The OEG lead fosters intense and open communication with the group members, in order to get information about their specific characteristics, understanding their ambitions, strengths and weaknesses. In doing so, the lead acquires the knowledge necessary to distribute the tasks according to the talent of the employees, their potential, and their preferences. The goal is to satisfy the projects requests in the most effective and efficient way, fully capitalizing on the group's human capital by assigning to each employee the tasks-portfolio best suitable.

The flexibility of the pools strategy constitutes a big advantage, since the employee does no longer have to focus on a single project. This factor enhances the possibilities the OEG lead has in assigning tasks to employees. In fact, the employee can support different projects in parallel, thus developing a broader spectrum of competencies. Still, this is not a must. Employees who have difficulties in staying tuned with more missions in parallel can request to focus on one single project.

The intense communication between the lead and the group members is also necessary for the lead to optimize the tasks distribution on a regular basis. In fact, some iteration is necessary in order to fine-tune the tasks portfolio for it to be challenging without overstressing or overloading the employee. Furthermore, the interests and the ambition of the employees change with time and their experience, and new and interesting tasks are coming from the

\footnotetext{
${ }^{* *}$ http://www.tuition.com.hk/psychology/i.htm
} 
project which naturally attract OEG group members and have to be assigned. Although the distribution of the tasks undergoes a constant optimization process, employees are assured that nothing is changed without listening to their opinion first. This leads to a feeling of working in a dynamic, yet predictable environment.

The scenario is made even more attractive by the courses and trainings offered by the DLR, which constitute for the employees a further possibility to extend their knowledge and to learn about new topics.

Keeping the Reiss theory in mind, the OEG lead allowed the sub-groups to work as self-managed teams, in order to satisfy the desire of independence of the engineers. The lead leaves as much autonomy as possible to the coordinators, and they are instructed to do the same with their engineers. The lead avoids acting in the area of competence of the sub-group coordinators; overruling of a coordinator decision is applied only in extreme cases and only after talking with the coordinator.

Another initiative which had a positive impact on employee motivation was to encourage creativity and innovation, as explained in the following chapter.

\section{Creativity and innovation}

Creativity is defined as "a mental characteristic that allows a person to think outside of the box, which results in innovative or different approaches to a particular task" "†'. Innovation is defined as "the process by which an idea or invention is translated into a good or service for which people will pay, or something that results from this process. Innovation involves deliberate application of information, imagination, and initiative in deriving greater or different value from resources, and encompasses all processes by which new ideas are generated and converted into useful products”

Since the main goal of the OEG is to use its resources in the most effective and efficient way, creativity and innovation are considered important aspects. On the other hand, mission operations must follow strict rules and validated procedures, with the purpose of avoiding mistakes that may lead to contingency situations and, in extreme cases, to the loss of a mission.

To encourage creativity and innovation maintaining at the same time the mentality necessary to perform mission operations in the most safe and reliable way, the OEG decided to create a "separated" environment, where the rules that are necessary to carry out the daily business do no longer apply.

For one afternoon every week, the operations engineers are free to engage themselves in topics different from their normal activities. A power and thermal engineer can, for example, read about team management, or have a talk about orbital dynamics with one of the experts working in the GSOC.

The group's members are also free to call meetings or brainstorming sessions to discuss their ideas, and organize presentations to get feedback from colleagues. It has been observed, that a lot of ideas which seem to be good at the beginning fail during this process, but the discussion with group members represents a sort of decision board rating the new proposal. This gives to the employees the feeling that their ideas have been heard and considered, which is not always the feeling when the idea is evaluated by a single person (typically the boss) and not further pursued in case of a negative opinion.

During these times reserved for creative work, mistakes are tolerated, and all ideas can be brought up for discussion, no matter how unconventional they might be. The people cultivate their creativity, and learn to deal with the resistance which is typical when proposals challenge routines which have been in place for a while. While discussing innovation, the group members exercise communication with other group members, sharing useful experiences that can help in supporting satellite missions.

The possibility to work on an idea that may improve the way operations are supported in the GSOC, together with the feeling that opinions can be expressed and are considered by colleagues are factors that have a positive effect on employee motivation. When talking about innovation, no pressure is applied in order to come up with results. In fact, it has been demonstrated that pressure and incentives in form of money do not help innovation. On the contrary, they may hinder it. Quoting the career analyst Dan Pink, for example: "If you want people to perform better, you reward them, right? [...] But that's not happening here. You've got an incentive designed to sharpen thinking and accelerate creativity, and it does just the opposite. It dulls thinking and blocks creativity".

When a group decides to foster innovation, the crucial role of the so-called "product champion", defined as "a senior individual or executive at a company that takes charge of furthering the internal development and external promotion of a certain good or service"»§ ${ }^{\S}$ has to be considered. In fact, the product champion significantly increases

\footnotetext{
${ }^{\dagger \dagger}$ http://www.businessdictionary.com/ definition/creativity.html

㧊 http://www.businessdictionary.com/ definition/innovation.html

$\S \S$ http://www.businessdictionary.com/definition/product-champion.html
} 
the probability of success of an innovation inside a market (in this case, the company). Therefore, the OEG lead takes care of presenting promising ideas to the department lead for further evaluation.

While fostering innovation, the OEG lead considers the possible conflicts with the defined group culture. In fact, corporate culture can "rule" the group, and the pressure to conform can become overbearingly high. Innovation has often to do with breaking rules, and a strong culture may therefore hinder it.

One may state that creativity does not come by request on a defined day. However, the establishment of the "free-thinking" afternoon is a constant reminder of the importance of innovation, and of the decision of the OEG to strive for performance at the leading edge.

\section{Achievements}

The mission operations team supporting a generic mission for Earth observation typically includes the following members: one AOCS engineer, one PTS engineer, one OBC/TMTC engineer, and one mission operations coordinator. For redundancy purposes, a backup should be provided for each position. In total, 8 engineers are required.

The OEG is currently offering a similar service for ten satellite projects, among them are two missions with two flying spacecrafts each. Two of these four satellites are far beyond their expected life time, and require demanding battery operation. One mission is currently close to launch, three projects are in the design phase, and four other projects are on "stand by".

Low management costs are achieved thanks to the establishment of a common group culture, which provides a basic understanding helping to minimize discussions and simplifying the internal decision processes.

The group engineers declared themselves satisfied with the way the OEG operates, and the tasks assigned. All perceived an increase of work efficiency and a decrease of uncertainty. Some reported difficulties in working on several projects in parallel. However, these problems are normally resolved by means of communication with the group lead. On the other hand, the multicolored task portfolio is highly appreciated.

Positive feedback was given about the campaign to harmonize the group culture, especially from new employees which had the chance to learn about the basics of the cooperation inside the OEG from the very beginning of their experience at the DLR. Particularly positive was the feedback about the time dedicated to innovation and creativity. According to the group members, this program enhanced employee motivation and improved communication between the different engineers.

\section{Conclusions and Further Developments}

This paper describes the mission operations support strategy implemented and applied since 2009 at the GSOC and focusing on the OEG. The structure of the group is described as well as the way engineers transitioned from supporting a single project to working for more projects in parallel. The advantages of this approach are presented, in terms of efficiency, job satisfaction, and employee motivation.

Together with these advantages, the establishment and maintenance of the new support strategy imply different challenges to be faced. The issue related to the role of the group lead and the possible conflicts with the project managers are presented, together with the possible problems of loyalty and identification.

The positive experience of the OEG convinced the GSOC management to extend the area of application of the pool strategy. At the moment, the operations engineers supporting communication mission have also been integrated in the group. The synergies between Earth observation and communication missions have still to be proven advantageous in practice. Nevertheless, the networking and the services offered by the OEG were considered as a strong argument in favor of the integration.

With the increasing size of the group, communication will become more challenging. This aspect is particularly important considering the crucial role an intense and open communication plays for the performance of the group. At the moment, several possibilities are under investigation to cope with this issue.

The improvement in terms of employee motivation and performance achieved after the harmonization of the OEG culture convinced the lead of the SEG to go through the same campaign. This campaign will take advantage from the experience made by the OEG lead. The harmonization of the SEG culture is also going to consider the OEG culture, with the purpose of achieving not only the maximum efficiency and effectiveness inside the SEG, but also the best possible cooperation between the SEG and the OEG. 


\section{Appendix A \\ The OEG culture}

The Operations Engineering Group’s main task is to support the satellite projects.

We are aware of the importance of this duty, and we fulfill it with particular focus on the quality of our output and the adherence to the due dates.

We are convinced the best results are achieved cooperating with the colleagues. We believe competition and rivalry must not be part of our Group's culture.

We support each project in a proactive way; we take care to report to the project manager about the possible improvement area we observe. On the other side, we are aware of the role of the project leader, and we are ready to accept his decisions.

Any Group's member knows that his performance and behavior has an impact not only on his reputation, but also on the reputation of the whole Group.

We consider the internal and external communication as a crucial factor to enable knowledge transfer, avoid single-points-of-failure, and improve the Group's achievements.

We take particular care of having a meetings concept which enables communication at operations subsystem level, as well as cross communication between the different subsystems.

The feedback culture is strongly encouraged. This is true inside the Group, but also in working together with the projects team. We provide (and expect from each other) a polite, honest and direct feedback.

We consider the development of the operations engineering as one of our top priorities. Our goal is to be on the leading edge! Therefore, we are constantly looking for management solutions enabling the implementation of new concepts, vision, and possible improvements. There is no idea which is too crazy to be discussed!

With this respect, we are always willing to expand our field of view, through the participation to conferences, seminar, and department meetings, as well as reading the information available on the DLR media instruments.

We commit to integrate any new Group member in the fastest way possible, and we expect the same commitment from any person joining our team. 


\title{
Appendix B \\ Acronym List
}

$\begin{array}{ll}\text { AOCS } & \text { Attitude and Orbit Control System } \\ \text { DLR } & \text { Deutsches Zentrum für Luft- und Rauhmfahrt } \\ \text { GSOC } & \text { German Space Operations Center } \\ \text { LEOP } & \text { Launch and Early Orbit Phase } \\ \text { OBC/TMTC } & \text { On-Board Computer / Telemetry and Telecommand } \\ \text { OEG } & \text { Operations Engineering Group } \\ \text { PMG } & \text { Project Management Group } \\ \text { PTS } & \text { Power and Thermal System } \\ \text { SEG } & \text { Software Engineering Group }\end{array}$

\section{Appendix C}

Glossary

\begin{abstract}
Spacecraft subsystem (or A part of the spacecraft system. Typical subsystems are the AOCS, the subsystem) OBC/TMTC, and the PTS

Space system The spacecraft
\end{abstract}

11

American Institute of Aeronautics and Astronautics 


\section{References}

${ }^{1}$ European Cooperation for Space Administration (ECSS), ECSS-E-ST-70C, Space Engineering, Ground systems and operations, ESA-ESTEC Requirements \& Standards division, 2008, Chap. 5.

${ }^{2}$ Houben, A., Frigge, C., Trinczek, R., Pongrats, H. J., Veränderung erfolgreich gestalten, C4 Consulting, 2007.

${ }^{3}$ Baglieri, E., Biffi, A., Coffetti, E., Ondoli, C., Pecchiari, N., Pilati, M., Poli, M., Sampietro, M., Organizzare e gestire progetti, Etas, 2004, Chap. 3.

${ }^{4}$ Pongratz, H. J. \& Trinczek, R., “BOHICA, Change zwischen Akzeptanz und Wiederstand,”, München, 2005

${ }^{5}$ Caldwell, B.. “Missteps, Miscues,” InformationWeek, June 20, 1994.

${ }^{6}$ Gross, T., Pascale, R., Athos, A., “the Reinvention Roller Coaster: Risking the Present for a Powerful Future,” Harvard Business Review, November-December 1993, pp 97-107.

${ }^{7}$ Kotter, J. P., Heskett, J. L., Corporate Culture and Performance, New York: Free Press, 1992.

${ }^{8}$ Chemers M., An integrative theory of leadership, Lawrence Erlbaum Associates, Publishers. ISBN 978-0-8058-2679-1, 1997.

${ }^{9}$ Kotterman, J., “Leadership Versus Management: What’s the Difference?,” Journal for Quality \& Participation.

${ }^{10}$ Kleinig, J, "Loyalty," The Stanford Encyclopedia of Philosophy, Aug 21, 2007, URL = < http://plato.stanford.edu/entries/loyalty/>

${ }^{12}$ Cheney, G., On the various changing meanings of organization membership: A field study of organizational identification, Communication Monographs, pp. 342-362.

${ }^{13}$ Pratt, M. G. (1998). “To be or not to be: central questions in organizational identification.” In Whetten, David A., and Paul C. Godfrey, Identity in organization, 171-207. Thousand Oaks: Sage.

${ }^{14}$ Cheney, G. and Tompkins, P. (1987). “Coming to Terms with Organizational Identification and Commitment,” Central States Speech Journal, 38/1, pp. 1-15.

${ }^{15}$ Tompkins, P., Apollo, Challenger, Columbia: The Decline of the Space Program, A Study in Organizational Communication, Roxbury Publishing Company: Los Angeles, 2005.

${ }^{16}$ Scott, C., Corman, S. and Cheney, G. (1998). "Development of a Structurational Model of Identification in the Organization, “ Communication Theory, 8/3, pp. 298-336.

${ }^{17}$ Cameron, K.S., Quinn, R.E., Diagnosis and Changing Organizational Culture, Addison-Wesley, 1999.

${ }^{18}$ McClelland, D. C.. Power: The inner experience. New York: Irvington, 1975.

${ }^{19}$ Herzberg, F., Mausner, B. \& Snyderman, B.B., The Motivation to Work, John Wiley, New York, 1959.

${ }^{20}$ Reiss, S., Who Am I? The 16 Basic Desires That Motivate Our Actions and Define Our Personalities, New York, 2000 